\title{
Methods of Coherent X-Ray Diffraction Imaging
}

\author{
P. A. Prosekov ${ }^{a, *}$, V. L. Nosik ${ }^{a, c}$, and A. E. Blagov ${ }^{b}$ \\ a Shubnikov Institute of Crystallography, Federal Scientific Research Centre “Crystallography and Photonics,” \\ Russian Academy of Sciences, Moscow, 119333 Russia \\ ${ }^{b}$ National Research Centre “Kurchatov Institute," Moscow, 123182 Russia \\ ${ }^{c}$ National Research Nuclear University MEPhI (Moscow Engineering Physics Institute), Moscow, 115409 Russia \\ *e-mail:prosekov@crys.ras.ru \\ Received June 10, 2021; revised June 10, 2021; accepted July 2, 2021
}

\begin{abstract}
Methods of coherent X-ray diffraction imaging of the spatial structure of noncrystalline objects and nanocrystals (nanostructures) are considered. Particular attention is paid to the methods of scanningbased coherent diffraction imaging (ptychography), visualization based on coherent surface scattering with application of correlation spectroscopy approaches, and specific features of visualization using X-ray freeelectron laser radiation. The corresponding data in the literature are analyzed to demonstrate the state of the art of the methods of coherent diffraction imaging and fields of their application.
\end{abstract}

DOI: $10.1134 / \mathrm{S} 1063774521060286$

\section{CONTENTS}

Introduction

1. Methods of Coherent X-Ray Diffraction Imaging

1.1. Coherence, Speckle Structure, CDI

1.2. Structure Reconstruction from CDI Data, Iterative Phase Retrieval Algorithms

2. Ptychography

3. Coherent X-Ray Surface Imaging

4. X-Ray Photon Correlation Spectroscopy and Speckle Spectroscopy

5. X-Ray Cross-Correlation Analysis

6. Specific Features of XFEL Imaging

Conclusions

\section{INTRODUCTION}

$\mathrm{X}$-ray crystallography has played an important role in many fields of science and technology and determined the direction of development of methods for studying the 3D structure of substances and materials on the atomic and nanoscale levels [1, 2].

Historically, an important role in the development of the aforementioned methods was played by the approaches of conventional crystallography based on $\mathrm{X}$-ray diffraction (XRD) analysis. Application of XRD to protein crystals led to great success in studying the structure and mechanisms of functioning of operation biological macromolecules and their ensembles, which served a basis for scientific and technological breakthrough in structural biology and biotechnolo- gies, as well as in various fields of genetics and medicine when developing new medicines.

In the Soviet Union a great contribution to the development of the XRD method and crystallography as a whole was made by Academician B.K. Vainshtein, who headed for many years the Shubnikov Institute of Crystallography of the Academy of Sciences of the Soviet Union. Studies on the crystallography of biomacromolecules began in his laboratory even in the 1950s [3-5]; crystals of some very important proteins were synthesized for the first time and their atomic structure was studied [6].

However, specifically the crystal structure imposes significant limitations on the preparation and choice of an object of study $[7,8]$, because many samples that are of interest for modern materials science, physics of nanosystems, chemistry, and biology, are poorly crystallized (an example is cell wall proteins, which are insoluble in water). Naturaly, the popularity of the crystallization approach was related to the insufficient intensity of X-ray sources, which hindered the study of the structure of noncrystalline and weakly ordered samples and the processes occurring in them.

In 1980 D. Sayre proposed to adapt crystallography methods for studying aperiodic objects in order to develop a new approach to imaging with a high spatial resolution based on coherent X-ray diffraction [9]. The history was repeated: the first diffraction pattern of a protein crystal was recorded in 1934 [10], but the first structures of globular proteins were identified much later, in 1958 [11]; the first observation of X-ray diffraction from an isolated object was reported in 
1987 [12], but an image was reconstructed from X-ray data only in 1999 [13].

Methods based on the use of coherence of electromagnetic radiation in the $\mathrm{X}$-ray range-coherent $\mathrm{X}$ ray diffraction imaging (coherent diffraction imaging (CDI) - - have been actively developed in the last decade. They provide amplitude- and phase-contrast imaging of an object studied and are aimed at determining the 3D structure of noncrystalline samples and nanocrystals (nanostructures) with a resolution that is theoretically restricted by only the diffraction limit [14]. Examples of such samples are various biological objects [15], including biological cells and viruses, and nanocrystallites of poorly crystallized macromolecules and their complexes. The development of CDI methods makes it possible to extend significantly the range of objects studied and, correspondingly, the range of scientific problems to be solved [16], including such fields of prime importance in Russia [17] as biotechnology, medicine and genetics, design of new functional (construction, composite, etc.) materials, and hybrid and nature-like technical systems (sensors, biosensors, etc.). A particular role in the development of CDI methods belongs to Mega Science facilities: synchrotron radiation (SR) sources of the fourth generation and X-ray free-electron lasers (XFELs) [18]. Along with the development of new SR sources, a number of programs have been started that are aimed at upgrading the functioning SR sources (KSRSKurchatov, ESRF-EBS [19, 20], PETRA IV [21, 22], APS [23], etc.) and creating new specialized stations on the functioning sources, designed for experiments on imaging micro- and nanoobjects using CDI methods (NanoMAX on the MAX IV source in Sweden, ID16A/B and ID10 on the ESRF source in France). To date, most of SR sources have almost $100 \%$ transverse coherence, and the upgrade of megafacilities is aimed mainly at reducing further the source emittance below $1 \mathrm{~nm}$, increasing brightness, and carrying out experiments with a pico- or femtosecond temporal resolution.

The development of CDI methods and techniques of analysis of coherent images of nanostructures is one of the problems of prime importance in the Russian Federation within the plan of creation of a series of megafacilities, including the SILA facility [24] (combination of a fourth-generation SR source and an X-ray free-electron laser).

The purpose of this review is to acquaint the reader with the modern CDI and coherent scattering methods, including CDI in the mode of elongated-object scanning (ptychography) based on coherent surface scattering, using correlation spectroscopy approaches and XFEL radiation. In view of the diversity of methods and approaches, their constant development and need for them, $100 \%$ coverage of the literature on this subject area appears impossible. In particular, holography and phase contrast methods are omitted; a review of their modern state of the art can be found in $[25,26]$.

\section{METHODS OF COHERENT X-RAY DIFFRACTION IMAGING}

One can arbitrarily divide all CDI versions into five main groups (see schematic diagrams in Fig. 1).

CDI in the plane-wave approximation (Fig. 1a) was presented for the first time in [9]. A coherent X-ray plane wave is incident on a sample, which is completely illuminated, and a two-dimensional diffraction pattern is recorded in the far-field zone (Fraunhofer diffraction). From the experimental point of view, important features of this geometry are relatively low sensitivity to vibrations and small sample displacements and possibility of carrying out single-shot experiments under conditions of short-term (e.g., pulsed) interaction of radiation with an object studied, including the case where this interaction leads to object structure degradation (damage) or destruction.

A schematic of X-ray ptychography is presented in Fig. 1b. In this case the linear sizes of sample studied exceed the transverse sizes of incident X-ray beam. The latter is generally formed using a pinhole or focusing coherent optics, which includes compound refractive lenses [27], planar compound refractive lenses [28], Fresnel zone plates [29-31] and a system of Kirkpatrick-Baez mirrors [32]. A ptychography experiment implies scanning over the 2D grid of extended object, with simultaneous detection of a series of diffraction patterns from partially overlapped regions of the sample studied.

The schematic of CDI in the Bragg geometry (Fig. 1c) is similar to that presented in Fig. 1a, but a diffraction pattern is recorded in the vicinity of Bragg reflection in the case of crystalline object (nanoscale structures, nanocrystals, etc.).

The essence of CDI in the Fresnel approximation (Fig. 1d) is as follows: a sample is installed before or behind the focal plane of Fresnel zone plate, after which a $2 \mathrm{D}$ diffraction pattern is recorded in the nearfield zone (Fresnel diffraction).

A schematic of CDI in the total external reflection (TER) geometry is shown in Fig. 1e: a sample is place on the surface of a substrate, which is oriented in the grazing incidence geometry close to TER conditions; a $2 \mathrm{D}$ diffraction pattern is recorded in the far-field zone in the vicinity of specularly reflected beam.

The CDI methods in the plane-wave approximation and Bragg geometry, as well as ptychography and their combinations, are being actively developed and demanded in studies of various micro- and nanostructures, as well as biological objects (using SR and XFEL). A number of researchers reported the use of CDI methods to image such biological objects as cells, organelles, bacteria [33-38], and some viruses [39, 40]. CDI makes it possible to determine the density of 

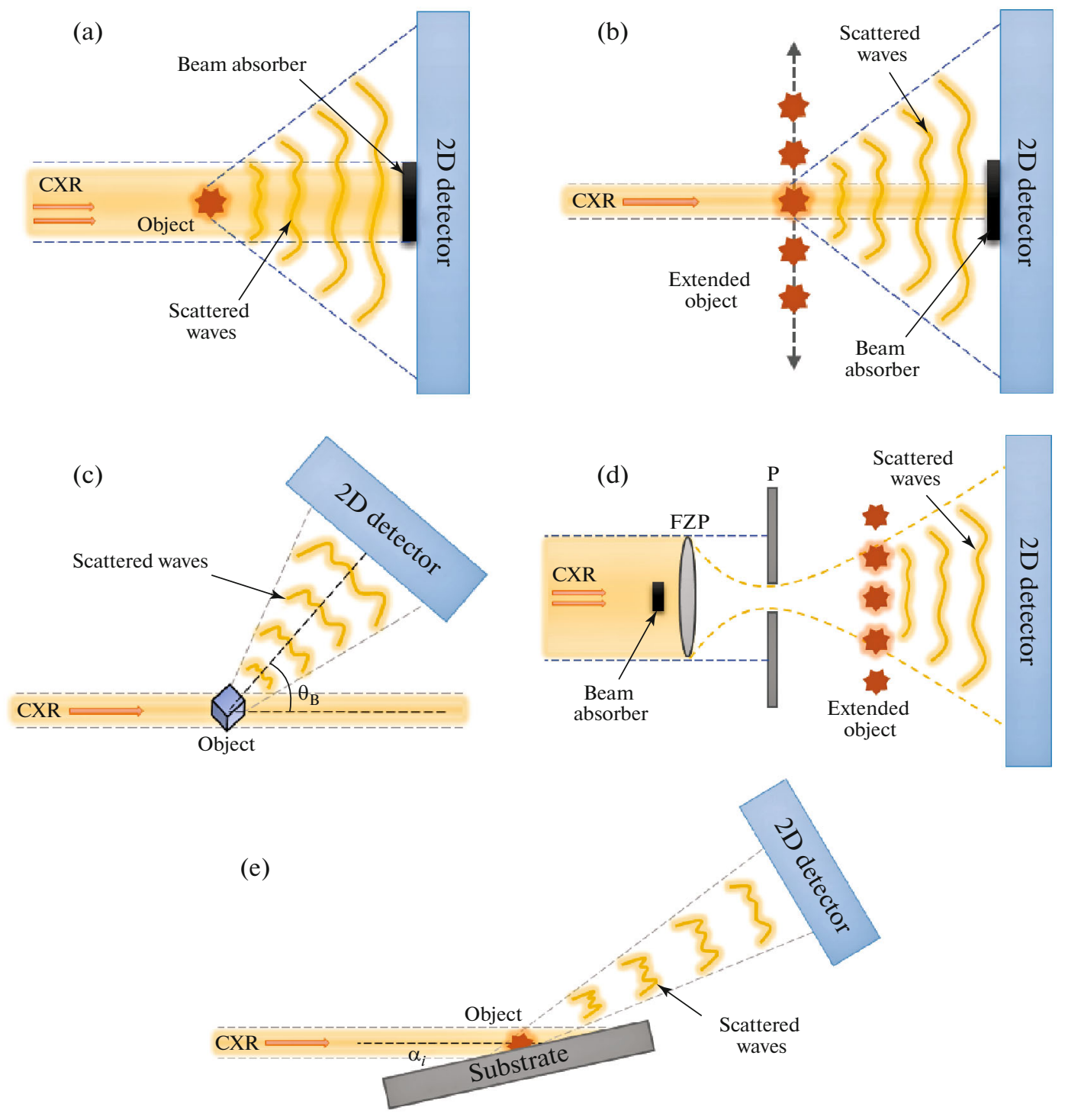

Fig. 1. Block diagrams of CDI versions: (a) plane-wave approximation, (b) X-ray ptychography, (c) Bragg geometry, (d) Fresnel approximation, and (e) TER geometry, with indication of (CXR) coherent $X$ rays, $\left(\theta_{B}\right)$ Bragg angle, (FZP) Fresnel zone plate, (P) pinhole, and $\left(\alpha_{i}\right)$ specular reflection critical angle of substrate. Irradiated regions ("asterisks") in (b) are shown non-overlapping for clarity [14].

material, based on which one can establish the position of different phases in material bulk.

In addition, CDI methods make it possible to perform chemical [41], elemental [42], and magnetic [43] nanomapping of complex (inhomogeneous, layered, and composite) materials. Resonance X-ray absorption at a photon energy specified for a given material of provides the so-called chemical contrast, in particular, due to the detection of fluorescence yield and application of X-ray electron spectroscopy for chemical analysis. Using polarized radiation, one can detect a magnetic contrast, which yields information about the distribution of magnetic moments and orientation of molecules in the sample. Here, the objects of study are amorphous and disordered structures, including glasses and polymers, as well as quantum dots, nanorods, and other nanostructures [44-46]. Some studies were devoted to the distribution of strains and stresses in the bulk of nanocrystals $[47,48]$ and imaging their shape [49].

A distinctive feature of the CDI approach in Fresnel approximation is the use of an incident wave with a specified wavefront curvature. It was shown in [50, 51] that, when a wave with a known (finite) curvature is incident on a sample, there is a single solution to the problem of structure reconstruction for the object studied. Williams et al. [52] reported the results of structure reconstruction for an aperiodic gold sample with a resolution of $24 \mathrm{~nm}$ by the CDI method in Fresnel approximation, using a zone plate with an external 
zone size of $50 \mathrm{~nm}$. An image of a standard sample with a resolution of $20 \mathrm{~nm}$ was reconstructed in a similar way in [53].

\subsection{Coherence, Speckle Structure, CDI}

The coherence of X-ray beam can be improved in two ways: decreasing the source sizes and increasing the distance from the source to the observation point. Despite the steady decrease in the source emittance, the electron-bunch size cannot be reduce to a point, because the use of beams with a low charge leads to radiation power loss, whereas the transverse size of high-charge beams is difficult to decrease because of the Coulomb repulsion. Therefore, the radiation in the far-field zone is used in practice, and its coherent part is selected using a system of slits (pinholes). This approach, although reducing the X-ray fluence on a sample, provides spatial filtering of incoherent modes.

Generally the coherent properties of X-ray beam are quantitatively characterized by the temporal (or longitudinal) and spatial (transverse) coherence lengths. The longitudinal coherence length $L_{1}$ is defined as the distance at which the phases of two harmonics with wavelengths of $\lambda$ and $\lambda+\Delta \lambda$ differ by $\pi$ :

$$
L_{1}=\frac{\lambda^{2}}{2 \Delta \lambda},
$$

and it can also be related to the spectral band $\Delta \lambda / \lambda$ of pulsed radiation.

The spatial (transverse) coherence is determined from the interference pattern in the case of diffraction from a system of two slits. For modern SR and XFEL sources the coherence length almost coincides with the transverse beam size and increases with a decrease in the source size $S$ and increase in the distance from the source to the observation point $R$ :

$$
L_{t}=\frac{\lambda R}{2 S} .
$$

For the SR sources of generation 3+ or higher, as well as for XFEL, the ratio of the total spatial coherence area to the entire beam cross section on a sample (i.e., after all transformations in the X-ray optical system) is $96-99 \%$; hence, these sources can be referred to as completely coherent.

When recording a diffraction pattern, a detector is placed in the far-field zone of Fraunhofer diffraction, where $\varepsilon=d^{2} / \lambda D \leqslant 1, \lambda$ is the wavelength, and $d$ is the transverse object size. Multiple scattering of X-ray pulses from a sample leads to the occurrence of local interference maxima in the form of speckles in the diffraction pattern. A speckle structure-an averaged interference pattern of the intensity distribution of coherent radiation diffracted (scattered) from the object studied-is formed from the speckle ensemble.

Note that the approaches based on correlation spectroscopy, in which is the speckle structures of coherent scattering patterns for weakly ordered and disordered systems, imaging at coherent surface scattering, and some other techniques are generally considered as a separate group. A general approach to the object structure reconstruction is the use of iterative phase reconstruction algorithms.

In the reciprocal space the size of a speckle obtained from an object (or its structural part) with a transverse size $d$ is given by the expression $\Delta q=2 \pi / d$, where $q$ is the wave vector. The minimum distance between speckles upon projection on the detector plane is determined as

$$
\Delta x=\frac{\Delta q}{k} D=\frac{\lambda D}{d} .
$$

According to the Kotel'nikov theorem (known as the Nyquist-Shannon sampling theorem in the foreign literature), to carry out of unambiguous reconstruction of the initial continuous signal with a limited spectrum from its discrete references, the pixel size $p$ should be less than half of the distance between speckles:

$$
p \leq \frac{\lambda D}{2 d} .
$$

If the size of the region occupied by an object is $N \times N$ pixels on a 2D grid, the number of known parameters in reciprocal space will be smaller by a factor of 2 , because only intensity is recorded. Therefore, one must consider an area in the image containing several times larger amount of information for the socalled sampling [54-56]. Sampling equal to 2 is the minimum necessary condition for successful object reconstruction.

Real-space sampling is also performed for the reconstructed object. With allowance for sampling and expression (3), the maximum spatial resolution of object (minimum size of object portion) is related to the detector $N p$, where $N$ is the linear number of detector pixels:

$$
\Delta d=\frac{\lambda D}{N p} .
$$

Typical distances between the sample and detector generally do not exceed several tens of meters, $p$ is 50$100 \mu \mathrm{m}$, and $N \sim 500-1000$.

\subsection{Structure Reconstruction from CDI Data, Iterative Phase Retrieval Algorithms}

The diffraction pattern obtained by CDI is in some sense a $2 \mathrm{D}$ projection of the Fourier transform of a real structure (electron density) of the sample studied on the detector surface. However, the reconstruction of sample structure via inverse Fourier transform of diffraction pattern is a nontrivial problem, and not only because of the limited detector size. Since each detector pixel records intensity (i.e., squared wave-field amplitude) rather than amplitude and phase, the total 
phase information appears to be lost. However, this is not quite so in the case of data analysis over the entire pixel ensemble.

This "phase problem," both in wave optics and in X-ray crystal optics [57], can be solved by fulfilling the following conditions: (i) when recording a diffraction pattern, the sampling criterion exceeds the value in correspondence with the Kotel'nikov theorem and (ii) in the absence of noise the size of recorded array of points in the diffraction pattern should exceed or be equal to the size of the array of points of reconstructed real structure. Then one can state (with some mathematical assumptions) that the phase information sufficient for reconstruction is contained (in the enciphered form) in the recorded image and can be extracted using special computational algorithms: iterative phase reconstruction algorithms.

Note that the spatial resolution of CDI methods is limited in theory mainly by the radiation wavelength and amounts to several tens of nanometers in X-ray range. However, the spatial resolution is limited in practice by many factors, from the brightness and coherence of the radiation source in use to the specific features of CDI scheme. For the modern SR sources of the third and fourth generations and XFEL, the resolution of CDI methods is in the range of $\sim 1-10 \mathrm{~nm}$; thus, CDI takes an intermediate position in this respect between the electron and X-ray microscopies.

An iterative phase reconstruction algorithm is a cyclic procedure based on fast Fourier transform of diffraction speckle structure, recorded by a detector with data modification (to solve the phase problem) at certain cycle steps $[56,58-62]$.

Currently, researchers use two efficient phase retrieval algorithms: Fienup (phase error reduction) algorithm and hybrid input-output algorithm [59, 60]; they both develop the Gerchberg approach, which was applied for the first time to solve the inverse problem in electron microscopy [58].

A common feature of all phase retrieval algorithms is the use of iterative transitions between the real and reciprocal spaces, which are performed via the fast Fourier transform over the so-called Fienup cycle [56]. The latter includes the following steps (Fig. 2):

(1) assignement of a random phase (initial assumption based on an a priori model) to the modulus of amplitude of experimentally obtained diffraction pattern and application of fast Fourier transform;

(2) superposition of boundary conditions (for example, approximate limitation of the object size, which can be estimated based on oversampling) on the image obtained in real space and application of direct fast Fourier transform;

(3) replacement of the amplitude of X-ray field strength in the obtained Fourier transform (in the reciprocal space) which the experimentally found amplitude and application of inverse fast Fourier transform;
(4) repetition of steps 2 and 3 (one iteration) up to the desired convergence level.

Convergence is estimated from the value of error, which is defined as the difference between the calculated and experimental amplitudes of the Fourier transform of object under study. Generally phase can be retrieved after $\sim 10^{2}-10^{3}$ iterations.

There are many versions of iterative methods (see review [62]). The most popular one is the guided hybrid input-output algorithm [63].

\section{PTYCHOGRAPHY}

One of the most significant limitations of CDI methods (both in the plane-wave approximation and in the Bragg geometry) is that only relatively small objects can be imaged; this limitation is related to the spatial coherence length of radiation (and beam sizes). A modification of the CDI method based on spatial scanning of extended objects, known as ptychography or scanning CDI (Fig. 1b), removes the aforementioned limitation and allows one to retrieve the object structure with a nanoscale spatial resolution $[64,65]$.

Ptychography originates from the pioneer study [66], where a technique of retrieving phase information about a crystalline object scanned by a coherent electron beam of finite sizes was described. This technique was developed in [67-69] for studying disordered objects, combined with iterative phase retrieval algorithms [70, 71], and demonstrated for the case of $\mathrm{X}$ rays [72]. Results of successful phase and amplitude retrieval for some objects (in particular, a test gold zone plate with a resolution of several tens of nanometers) were reported in $[73,74]$. The possibility of imaging the structure of objects with sizes exceeding the transverse sizes of coherent beam makes this method promising for studying condensed matter [75-77] and biological systems [78, 79].

The method implies sample scanning (displacing the illumination spot on the sample surface, formed by a coherent focused or pinhole-limited beam), with overlap of illuminated areas and simultaneous detection of an array of diffraction patterns. A set of diffraction patterns from different but partially overlapping areas allows one to reconstruct successively a $2 \mathrm{D}$ or $3 \mathrm{D}$ image of object structure with application of iterative algorithms.

Scanning with a focused beam makes it possible to obtain not only structural contrast by also additional chemical contrast (due to the X-ray fluorescence) with a high elemental sensitivity.

Ptychography is generally implemented as a $2 \mathrm{D}$ method, which provides phase images due to the difference in the projections of optical path length in sample. To ensure these conditions, the sample must be optically thin. Then the wavefront passing through the sample can be exactly modeled by multiplying the distribution functions of the incident beam intensity 


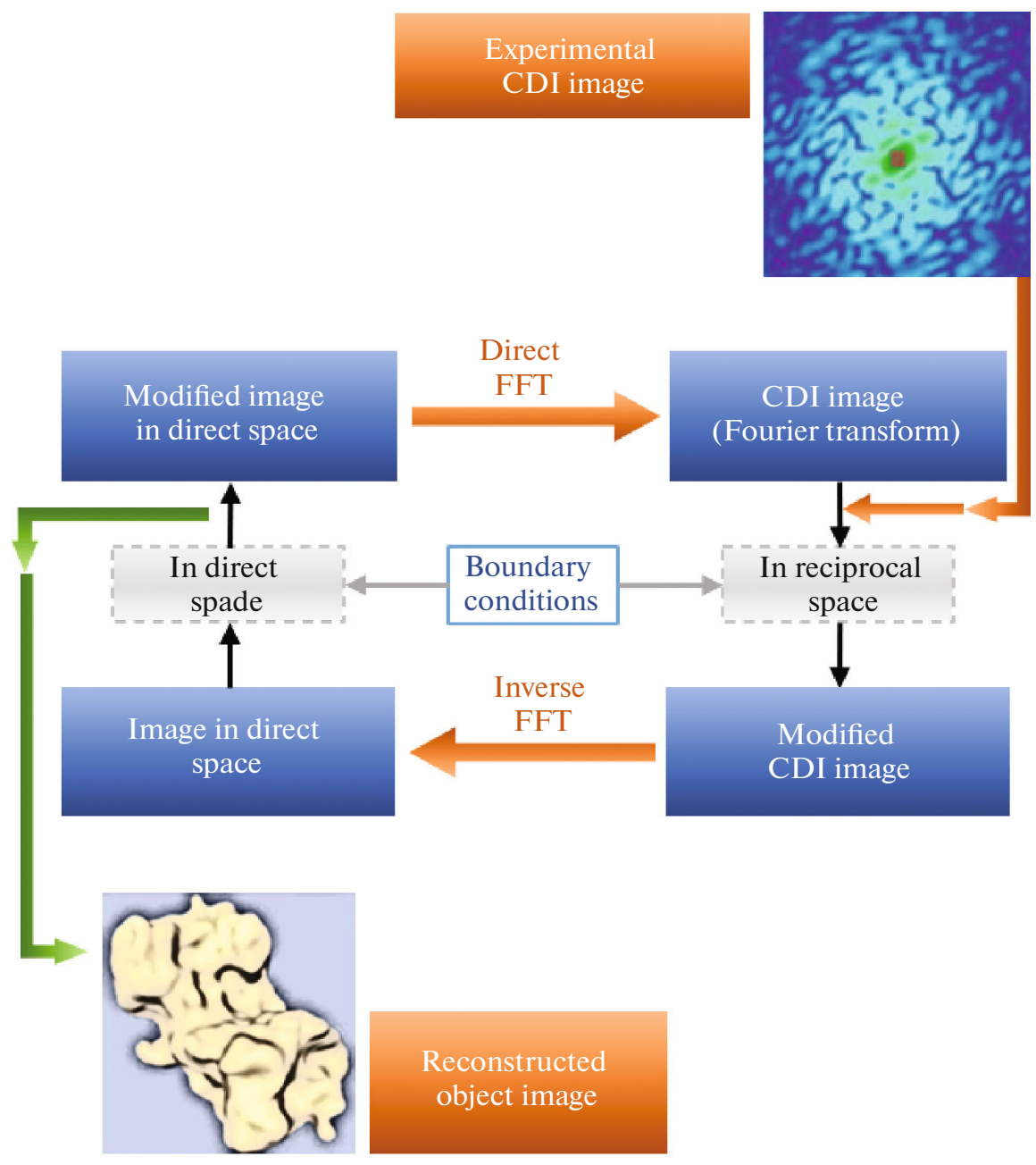

Fig. 2. Block diagram illustrating the procedure of image reconstruction for an object studied from the CDI data using an iterative phase retrieval algorithm with fast Fourier transform (FFT) [14].

and the sample transfer function. The limiting value of sample thickness for this approximation was determined theoretically in $[73,80]$ and then refined using numerical calculations; as a result, the following relation was obtained [81]:

$$
T \leq \frac{5.2 \Delta r^{2}}{\lambda}
$$

where $T$ is the sample thickness, $\Delta r$ is the image resolution, and $\lambda$ is the illumination wavelength. The corresponding Fresnel number is no less than 0.2.

In contrast to the standard image reconstruction algorithms (error reduction, hybrid input-output, etc.), ptychography implies simultaneous processing of several diffraction patterns from overlapping regions of the sample. Sufficient overlap of patterns makes it possible to solve the phase problem and reconstruct the sample structure image. Reconstruction is performed using a test function (function of the projection of incident radiation beam on the plane of object studied) $P(r)$ and the object function $O(r)$, which characterizes the sample studied $[64,65]$. Then, in the presence of a relative displacement $r_{i}$ between the sample and test function, the resulting wave field behind the sample (recorded in the far-field zone) $\Psi_{i}(r)$ is

$$
\Psi_{i}(r)=P(r) O\left(r-r_{i}\right) .
$$

The essence of ptychographic iterative engine for image reconstruction consists is successive refinement of the object function $O(r)$ by applying direct and inverse fast Fourier transforms, with transition to every new test taking into account the information about the previous one (Fig. 3). In addition, an extended ptychographic iterative engine was developed, in which, along with the object function $O(r)$, the test function $P(r)$, which may be initially unknown, is also refined.

The ptychographic approach can be efficiently applied in combination with other imaging methods. For example, in the so-called 3D ptychographic tomographic transforms are additionally used to obtain 3D images with a high spatial resolution. In 


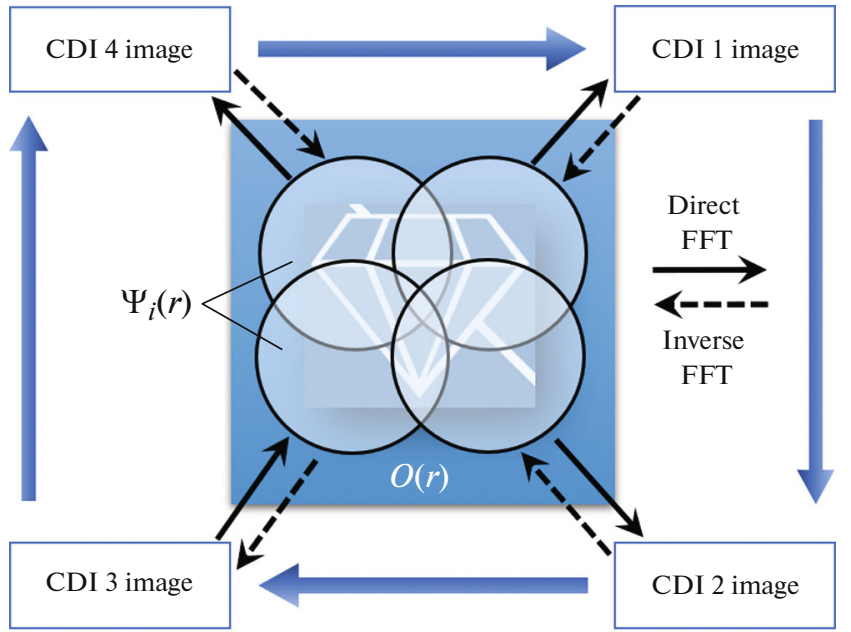

Fig. 3. Schematic diagram of iterative phase retrieval algorithm. Gray arrows indicate successive transitions between CDI images, with renewal of information about the object. Black arrows indicate direct (solid) and inverse (dashed) fast Fourier transforms [64].

simple cases of ptychographic tomography (Fig. 4a) a $3 \mathrm{D}$ image is obtained rotating a sample by $180^{\circ}$ with a small step, and a ptychographic projection is recorded for each angle. When all imaging projections for each angle are reconstructed using ptychography iterative algorithms, they are processed using standard tomographic programs, for example, the inverse-projection algorithm [82]. As a result, a 3D image of the object studied is formed.

Multi-slice ptychography is applied in more complex situations, when the sample thickness exceeds the multiplicative approximation limit [83]. Within the multi-slice model a thick sample is considered as a set of thin cuts, for each of which multiplicative approximation can be used (Fig. 4b). The experimental scanning process is presented in Fig. 4c. The wave field in the detector plane can be calculated using the calculation block diagram presented in Fig. 4d.

To reconstruct the image, the calculated wave field at the output is first reconsidered with correction of phases and replacement of intensities, and then the calculation block diagram order is changed to opposite (inverse). Each of thin cuts can be reconstructed using standard algorithms [85] for a single cut [86].

The additional advantages of multi-slice ptychography and ptychographic tomography are in favor of their combination, in which the 3D information obtained by multi-slice ptychography is used to reduce the number of angular measurements (and the scan time). At the same time, this combined method of
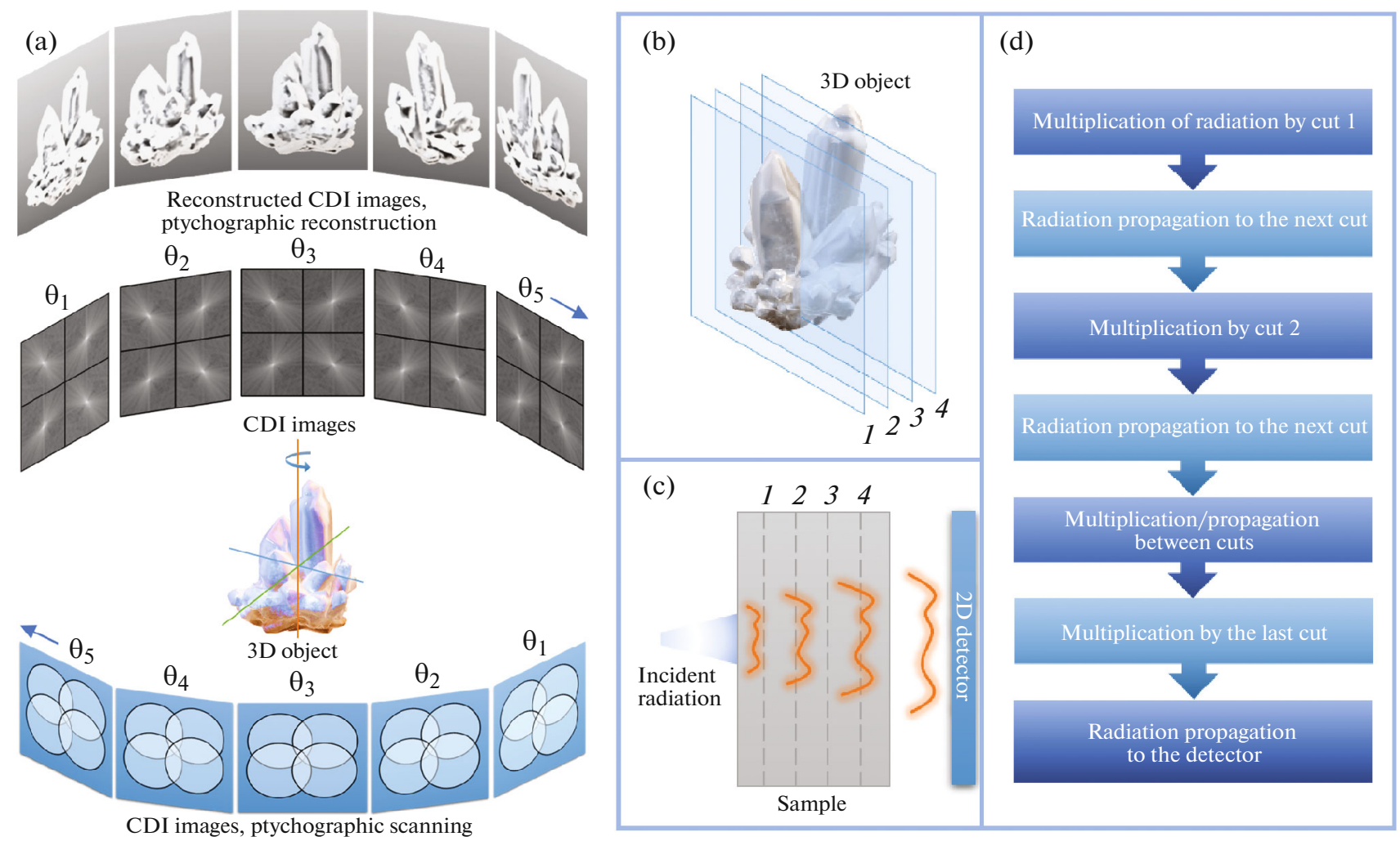

Fig. 4. Schematic diagram of 3D ptychography: (a) block diagram of ptychographic tomography; (b) division of sample into a series of thin cuts (layers), when the sample thickness exceeds the imaging depth in single-layer ptychography; (c) radiation propagation in a sample; and (d) block diagram of multistage computational model [84]. 
multi-slice ptychographic tomography [84] can be used for thicker samples with conservation of high resolution.

\section{COHERENT X-RAY SURFACE IMAGING}

The technique of coherent imaging of surface scattering is a modification of coherent scattering method. It allows one to study the 3D structure of surfaces, interfaces, and ordered and weakly ordered objects on the surface and in the bulk of material.

This method was proposed for the first time in 2012 [87]. Similar approaches were considered previously in $[88,89]$; however, without a possibility of reconstructing the $3 \mathrm{D}$ structure. The experiments reported in [87] were performed on the third-generation SR source APS (Advanced Photon Source). Since the coherent radiation intensity was insufficient, and the detector could not be installed at a necessary distance from the sample, the resolution of the reconstructed structure of the object studied in one of the directions was more than $500 \mathrm{~nm}$. It was suggested that the use of a fourth-generation SR source and optimization of the schematic of the experiment on coherent imaging of surface scattering will make it possible to reach a nanoscale spatial resolution. However, even now a resolution of $40 \times 80 \mathrm{~nm}$ in the surface plane and a subnanometer in the normal direction was obtained for "desktop" sources, generating radiation in the extreme UV (EUV) range стигнуто [90]. Thus, EUV X-ray ptychography has a resolution comparable with the record values for scanning electron microscopy but, in contrast to the latter, is not restricted to samples with conducting surfaces.

The essence of the method of coherent imaging of surface scattering is that a scattering pattern is recorded in the TER geometry in the vicinity of critical angle $\alpha_{i}$ (Fig. 1e). In the grazing incidence geometry surface scattering is extremely sensitive to the electron density distribution both in the lateral direction (along the sample surface) and along the normal [9193], due to which coherent scattering with a high signal-to-noise ratio and short recording time can be formed [87]. Combination of this method with ptychography and photon correlation spectroscopy will make it possible to scan in the aforementioned mode objects with sizes exceeding the transverse sizes of incident X-ray beam and gain information with a temporal resolution characteristic of correlation methods.

\section{X-RAY PHOTON CORRELATION SPECTROSCOPY AND SPECKLE SPECTROSCOPY}

X-ray photon correlation spectroscopy (XPCS), or intensity fluctuation spectroscopy [94-96], is based on analysis of the time evolution of CDI diffraction pattern and speckle structures. It is generally applied when studying weakly ordered and disordered objects, such as colloidal suspensions [97-99], mixtures of homopolymers [100], block copolymer micelles and vesicles [101, 102], nanoparticles in supercooled liquids [103], and antiferromagnets [104]. The standard scheme of XPCS experiment coincides with the scheme presented in Fig. 1a. XPCS is also actively used when studying the dynamics (Brownian motion) of metal nanoparticles in solutions, charge density waves, critical fluctuations at order-disorder phase transitions in amorphous alloys, and conformational dynamics of vitrified polymers [105-110].

XPCS makes it possible to study the structural dynamics in a sample from the intensity fluctuations in a recorded speckle structure, observe low-frequency dynamic processes, and visualize density fluctuation at distance of several nanometers [111].

In XPCS experiments information is derived from the data on intensity fluctuations using normalized second-order autocorrelation intensity function $g_{2}(q, t)$ :

$$
g_{2}(q, t)=\frac{\left\langle I\left(q, t^{\prime}\right) I\left(q, t+t^{\prime}\right)\right\rangle}{\left\langle I\left(q, t^{\prime}\right)\right\rangle^{2}},
$$

where $I(q, t)$ is the scattering intensity corresponding to scattering vector $q$ at an instant $t$ and angular brackets indicate averaging over ensemble (over measurement time). Averaging is performed over all intensity values, pixels of the scattering pattern speckle structure, and time as follows: it is suggested that $K$ successive speckle patterns with a frame frequency $f_{r}$ are recorded by an area detector with $N$ pixels, which correspond to scattering vector $q$. Then $g_{2}(q, t)$ can be written as

$$
\begin{gathered}
g_{2}\left(q, t=k^{\prime} \Delta t\right) \\
=\frac{\frac{1}{N\left(K-k^{\prime}\right)} \sum_{n=1}^{N} \sum_{k=1}^{K-k^{\prime}} S_{n, k} S_{n, k+k^{\prime}}}{\left(\frac{1}{N\left(K-k^{\prime}\right)} \sum_{n=1}^{N} \sum_{k=1}^{K-k^{\prime}} S_{n, k}\right)\left(\frac{1}{N k^{\prime}} \sum_{n=1}^{N} \sum_{k=K-k^{\prime}+1}^{K} S_{n, k}\right)},
\end{gathered}
$$

where $\Delta t=1 / f_{r}$ and $S_{n, k}$ is the scattering intensity in the $n$th pixel of $k$ th image after subtracting the averaged dark noise of detector. In the case of partially coherent radiation the function $g_{2}(q, t)$ is related to the normalized first-order autocorrelation function $g_{1}(q, t)$ by the Siegert relation [112]:

$$
g_{2}(q, t)=1+\beta\left|g_{1}(q, t)\right|^{2},
$$

where $\beta$ is the degree of coherence, which is varied from zero to unity.

Due to the analysis of autocorrelation function, XPCS makes it possible to determine the temporal characteristics of the Brownian motion of the system and relaxation of atoms/molecules in the sample, i.e., to determine the free-diffusion coefficients (constants) $D_{0}$, rms displacements of particles, and relaxation rates. 
XPCS is efficiently applied in studies devoted to aging, delayed deposition of particles in various colloidal systems, and formation of percolation sets of particles [113]. Time evolution (aging) can be quantitatively estimated using the autocorrelation function with the aid of "equal-age cuts" obtained from 2D autocorrelation functions. In contrast to the case of simple Brownian motion, when correlation functions can be presented in the form of simple exponentially decreasing functions, in this case necessary to use the Kohlrausch-Williams-Watt approximation:

$$
g^{(2)}(t)=\beta \exp \left(-\left(\frac{t}{\tau}\right)^{\gamma}\right)+1,
$$

where $\tau$ is the relaxation time (the relaxation rate is $\Gamma=1 / \tau)$ and $\gamma$ is the exponential factor.

$\mathrm{X}$-ray speckle spectroscopy (or speckle visibility spectroscopy), which based on quantitative analysis of the contrast of interference maxima in a speckle pattern and speckle visibility $v(q, t)$, has been developed as a method alternative to XPCS. X-ray speckle visibility spectroscopy yields the same information as XPCS but at times shorter than frame change period in the detector [114-118]. This method is especially demanded for in the case of XFEL; it opens ways for detecting ultrafast dynamics at the atomic level by analyzing the visibility of speckle patterns obtained using a pair of femtosecond X-ray pulses (pump and probe) with a relative time delay (from several femtoseconds to several nanoseconds) determining the "exposure time" [119-125].

The $v(q, t)$ value for a specified speckle pattern is determined by the formula

$$
v(q, t)=\frac{\left\langle S^{2}(q, t)\right\rangle-\langle S(q, t)\rangle^{2}}{\langle S(q, t)\rangle^{2}},
$$

where $S(q, t)$ is the scattering intensity determined for the exposure time $T\left(S(q, t)=\int_{0}^{T} I(q, t) d t\right)$. As well as in XPCS, averaging over ensemble in (12) is averaging over pixels and time: we assume the exposure time of successive images to be $T$; then the $v(q, t)$ value for the speckle structure is calculated as

$$
v(q, t)=\frac{\frac{1}{N K-1} \sum_{n=1}^{N} \sum_{k=1}^{K}\left(S_{n, k}-\frac{1}{N K} \sum_{n=1}^{N} \sum_{k=1}^{K} S_{n, k}\right)^{2}}{\left(\frac{1}{N K} \sum_{n=1}^{N} \sum_{k=1}^{K} S_{n, k}\right)^{2}} .
$$

One can show that $v(q, t)$ is related to $g_{1}(q, t)$ by the formula

$$
v(q, t)=2 \beta / T \int_{0}^{T}(1-t / T)|f(q, t)|^{2} d t .
$$

\section{X-RAY CROSS-CORRELATION ANALYSIS}

$\mathrm{X}$-ray cross-correlation analysis (XCCA) is based on the analysis of a speckle structure using CDI; it allows one to investigate the structure of individual particles, as well as disordered and partially ordered systems, scheme as alloys or colloidal glasses. In the standard scheme of XCCA experiment (Fig. 1a) correlations of scattered-field intensity in the reciprocal space, $I(\mathbf{q}, t)$, are sought for two chosen scattering vectors $\mathbf{q}$ at different instants, $t_{1}$ and $t_{2}$ :

$$
C\left(\mathbf{q}_{1}, \mathbf{q}_{2}, t_{1}, t_{2}\right) \sim I\left(\mathbf{q}_{1}, t_{1}\right) I\left(\mathbf{q}_{2}, t_{1}\right),
$$

where $C\left(\mathbf{q}_{1}, \mathbf{q}_{2}, t_{1}, t_{2}\right)$ is the cross-correlation function of intensity.

The concept of XCCA (or fluctuation scattering) was proposed for the first time by Kam in 1977 as a tool for determining the structure of individual macromolecules in solution [126] using visible light. The instantaneous particle distribution is fixed if the scattering by identical unoriented particles is recorded for a time shorter than the time necessary for their reorientation. A cross-correlation analysis of the angular variations in intensity on the detector area, supplemented with an analysis of variations in the radial direction (similarly to the small-angle $\mathrm{X}$-ray scattering method), makes it possible to reconstruct information about structure particles. Then this approach was developed by Clark for studying the local order in condensed phases for 2D systems (particle monolayer $\sim 230 \mathrm{~nm}$ in size) [127] and spatial correlation of particles in 3D systems (in colloidal liquid) [128].

The success in the development of X-ray and SR sources made it possible to apply the cross-correlation approach in experiments with coherent X-ray scattering. One of the first XCCA studies showed the possibility of revealing hidden symmetry (related to the local structure of amorphous sample) in closely packed colloidal glasses [129]. The theory of the XCCA method and the corresponding computer simulation algorithms were described in [130-133].

Two main directions can be selected in XCCA studies: (i) analysis of the structural features of weakly ordered systems and (ii) determination of the structure of single particles.

In the case of disordered systems, XCCA is used to study the local order and structure of ensembles of identical particles in colloidal 2D and 3D systems (in particular, under pressure [134]), analyze correlations in thin colloidal films in small-angle X-ray scattering geometry [135], determine quantitatively the ordering and bonds in liquid crystals [136], studying the structure of nanocrystalline superlattices [137], detect structural inhomogeneities in polymers [138], and investigate the local order and symmetry in magnetic domains [139].

Concerning the study of single particles, the XCCA method has been adapted so as to obtain structural information (crystallographic structure, size, and 
shape of particles); it is being widely applied for modern SR sources and XFEL. An XFEL with ultrashort (femtosecond) X-ray pulses of high brightness provides instantaneous "images" of randomly oriented particles.

Within the first approach the cross-correlation function is used as a limitation in the iterative phase retrieval algorithm. With the aid of this approach, the structure of single polystyrene dimers was determined on the XFEL LCLS (Linac Coherent Light Source) in [140] and the structure of 2D gold nanoparticles was established on the SR source SLS (Swiss Light Source) in [141]. The second approach is based on direct fitting (approximation) of real-structure model with allowance for the cross-correlation function in correspondence with the experimentally obtained scattering patterns.

Note that XCCA makes it possible to perform timeresolved measurements, which is important for understanding the processes of structure formation and crystallization and mechanisms of phase transitions. The form of cross-correlation function can be chosen in dependence of the purpose and experimentally determined parameters. For example, when studying the local order in static colloidal dispersions this function is determined via angular intensity correlations in polar coordinates with a constant modulus of wave vector $\mathbf{q}\left(\left|\mathbf{q}_{1}\right|=\left|\mathbf{q}_{2}\right|=q\right)$ :

$$
C(q, \Delta \varphi)=\frac{\langle I(q, \varphi) I(q, \varphi+\Delta \varphi)\rangle_{\varphi}-\langle I(q, \varphi)\rangle_{\varphi}^{2}}{\langle I(q, \varphi)\rangle_{\varphi}^{2}} .
$$

The scattering vector $\mathbf{q}$ lies in the plane oriented perpendicular to the incident beam; $\Delta \varphi$ is the angular difference between the scattering vectors $\mathbf{q}_{1}$ and $\mathbf{q}_{2}$, which takes values in range from zero to $2 \pi$ (Fig. 5a). The operator $\langle\ldots\rangle_{\varphi}$ indicates averaging over angle $\varphi$ along a ring of radius $q$.

Expansion of the correlation function $C(q, \Delta \varphi)$ in a Fourier series in $\Delta \varphi$ allows one to study in detail the local orientational order of a sample proceeding from the properties of function

$$
C(q, \Delta \varphi)=\sum_{l=-\infty}^{\infty} C_{l}(q) \exp (i l \Delta \varphi),
$$

with Fourier coefficients

$$
C_{l}(q)=\frac{1}{2 \pi} \int_{0}^{2 \pi} C(q, \Delta \varphi) \exp (-i l \Delta \varphi) d \Delta \varphi .
$$

The relationship between the Fourier coefficients $C_{l}(q)$ and structural orientational order can be illustrated by an example of a 2D system. Let the system contain five arbitrarily oriented hexagonal clusters of spherical particles (Fig. 5b). The calculated scattering pattern is presented in Fig. 5c. The Fourier coefficients $C_{l}(q)$ of the correlation function $C(q, \Delta \varphi)$, obtained from the calculated scattering pattern, are presented as a function of $l$ components in Fig. $5 \mathrm{~d}$. One can see the dominance of the Fourier coefficients with $l=6$ and 12, which reflect the hexagonal structural symmetry of the system.

\section{SPECIFIC FEATURES OF XFEL IMAGING}

The enhanced brightness [143] and time structure with specific 100 fs pulse is a characteristic feature of XFEL, which casts a new look at the possibilities of $\mathrm{X}$-ray time-resolved experiment. The XFEL provides a transition to samples of new types: nanoparticles and individual biomolecules [144, 145], in particular, in commercial-crystallography approaches [146]. New terms arose: megahertz $X$-ray diffraction $[147,148]$ and imaging [149]. Samples of this type do not call for crystallization [150], which is necessary when using SR [151].

However, the use of pulsed $\mathrm{X}$ rays gives rise to new problems, the most important of which are the control of wavefront distortions [152, 153], which may affects significantly the interaction of intense radiation with material [154]; sample destruction; and negative influence of XFEL radiation on the X-ray optics [155, 156].

In view of the more stringent requirements to the $\mathrm{X}$-ray optics radiation resistance, silicon gradually gives way to diamond (for example, as a material of Fresnel zone plates [157]) and beryllium compounds (for compound refractive lenses [158]). Note that the sample destruction with a characteristic time of $10-$ $50 \mathrm{fs}$ led to the development of the concept of diffraction pattern fixation "up to the instant of sample destruction" [159].

One of the bottlenecks of time-resolved X-ray experiments is X-ray detectors [160]. The extremely high frequency of modern multipixel $2 \mathrm{D}$ detectors is limited by a value of $5 \mathrm{MHz}$ for the AGIPD detector (which is being constantly upgraded), which corresponds to a frame change period of $200 \mathrm{~ns}$. Schemes of splitting the initial beam into two beams using a system of crystals and joint use of X-ray and optical laser radiations are active developed to improve the temporal resolution. In the former case a series of successive reflections leads to wavefront distortions, whereas in the latter case serious problems with synchronization of X-ray and light pulses arise.

Since the beginning of active operation of European XFEL stations new experimental schemes of $\mathrm{X}$-ray imaging have been proposed; the $\mathrm{X}$-ray optics is being upgraded as well. For example, the experimental station for materials imaging and dynamics MID on the European XFEL [144], which was put into operation in the end of 2018, makes it possible to study nanostructured materials, liquids, and soft and solid condensed media. Along with the tool for studying materials with high energy density (HED), MID uses radiation from an undulator SASE-2 with a photon energy of 5-25 keV, which provides up to $10^{12}$ pho- 

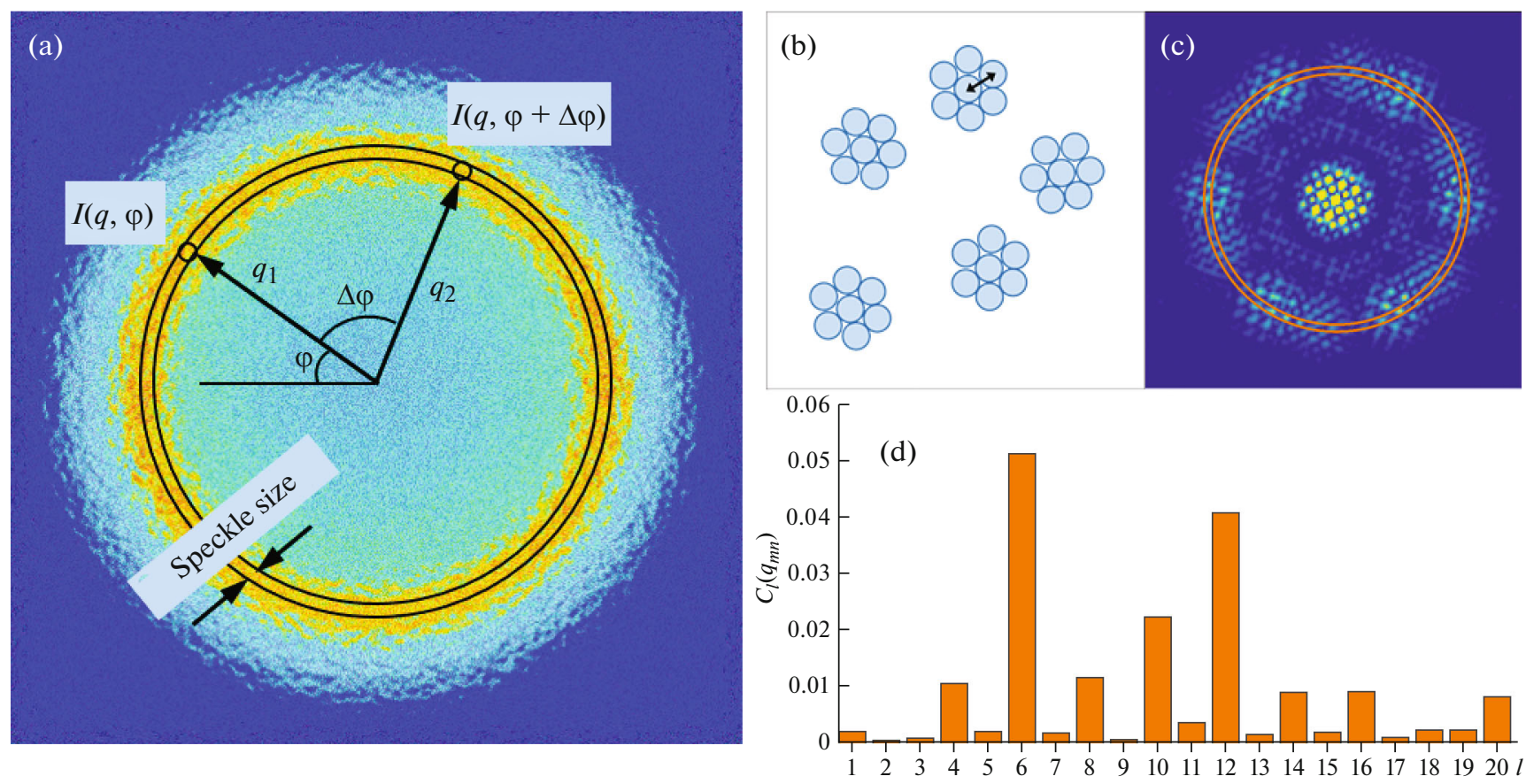

Fig. 5. (a) Determination of the parameters of cross-correlation function $C(q, \Delta \varphi)$. (b) XCCA on a model system from five arbitrarily oriented hexagonal clusters of spherical particles; the distance $r_{n n}$ between particles is shown by an arrow. (c) Calculation of coherent scattering from a given structure. (d) Fourier coefficients $C_{l}\left(q_{m n}\right)$ as a function of $l$ components, calculated for $q_{m n}=$ $2 \pi / r_{m n}[142]$.

tons/pulse and up to 3000 pulses/s. The X-ray splitand-delay line based on a series of diffraction reflections for the MID station was described in [161]; new concepts of improving stability and simplifying equalization of this line have been proposed recently $[162,163]$.

The imaging based on propagation in the near field (in particular near-field holography and its implementation with a high resolution in the conical-beam geometry) may ensure full-scale study of the sample dynamics under the action of a single XFEL pulse [155]. The problem of fluctuating wavefront is solved by sample the configuration space of SASE source pulses before actual image recording with subsequent analysis of the main component. In the case of European XFEL MID [155], an experiment on near-field holography with a temporal resolution is performed using aberration-corrected nanofocusing compound refractive lenses. The dynamics of the microfluidic jet used for supplying a sample is additionally analyzed (Fig. 6). Imaging in the pump-and-probe mode is implemented using an IR pulsed laser, whose radiation induces cavitation and explosion processes in the jet.

The pulsed character of XFEL radiation solves two main problems of CDI based on SR sources. First, images are not difused because of the sample motion (theoretically, the temporal resolution limit is determined by the pulse width: $100 \mathrm{fs})$. Second, the XFEL coherent radiation flux exceeds many orders of magni- tude that for a single SR pulse, due to which the requirement to the particle size and $\mathrm{X}$-ray optics as a whole become less stringent.

Phase-sensitive methods become even more promising, because the phase shift of refractive index for hard X rays may be several orders of magnitude larger than the change in the absorption coefficient. The use of the phase shift is especially important for biomaterials, which are based on light elements with a small number of electrons $Z$. However, only few from many available CDI methods are currently compatible with XFEL single-pulse imaging. In particular, ptychography [72] for nanoobjects and its interferometric analog at the meso- and macroscopic levels [164] cannot be implemented in full measure using single pulses. Despite the fact that the potential of ptychography was demonstrated for static samples [165], while the image processing can be parallelized to some extent [166], current experiments are limited to demonstrations of the possibilities of coherent diffraction imaging [13] and analysis of the structure of small biological particles, such as bacteria or viruses $[167,168]$. For example, even for eukaryotic cells more than $5 \mu \mathrm{m}$ in diameter the necessary requirements cannot be fulfilled because of the excess definiteness of image speckle structure.

The possibilities of near-field holography were also demonstrated for the images obtained using XFEL radiation $[169,170]$, but the problems with intensity 


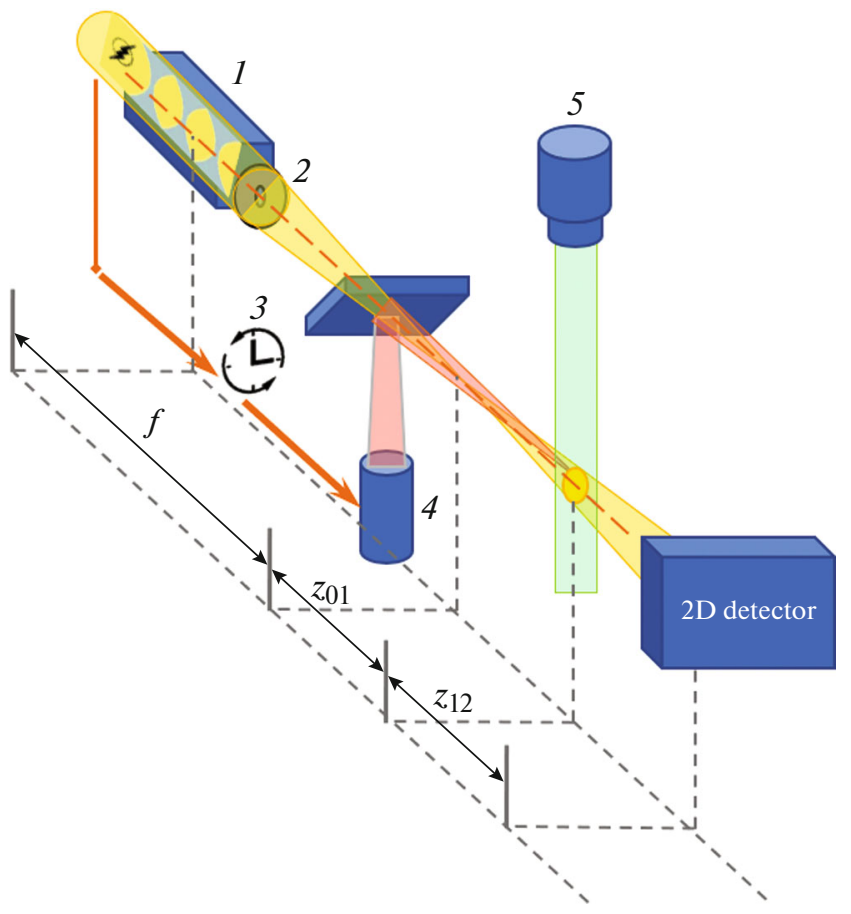

Fig. 6. Experimental scheme of European XFEL MID. XFEL radiation from SASE2 undulator is collimated by a set of compound refractive lenses 1 with a focal length $f$. Phase plate 2 is used to correct optical system aberrations, and delay generator 3 serves to trigger pump laser 4 at the instant of XFEL pulse arrival. Microfluidic jet 5 is located at a defocusing distance $z_{01}=271.3 \mathrm{~mm}$. The detector is installed at a distance of $z_{12}=9670 \mathrm{~mm}$ from the jet [155].

fluctuations due to the random character of photon generation in XFEL (in essence, shot noise amplification) and, correspondingly, with phase retrieval are not solved completely yet.

Note that the high brightness of XFEL makes it possible to implement previously inaccessible methods of diffraction pattern imaging using photoelectrons [171], methods of nonlinear X-ray physics [172], and new techniques of radiation polarization control [173].

\section{CONCLUSIONS}

The methods of coherent diffraction imaging considered in this review were elaborated and are being actively developed because new SR sources of the 3+ and fourth generations and XFEL are being put into operation. These sources, due to their brightness, small emittance, and temporal structure open up new horizons for X-ray diffraction studies and materials science. Despite the fact that the spatial resolution these methods $(\sim 1-10 \mathrm{~nm})$ cannot yet be compared to that of electron microscopy analogues (characterized by an angstrom resolution), the nondestructive effect of radiation from these sources and their exceptional brightness give grounds to expect active development of specifically non-diffraction X-ray methods and expansion of the range of noncrystalline objects of study.

To conclude, we should some specific features of CDI methods.

(1) In view of the use of pulsed sources and application of phase-sensitive methods, particular attention is paid to the control of the wavefront and its distortions.

(2) The general direction of studies shifts towards noncrystalline objects (nanoparticles and various biological objects, such as DNA, proteins, and biomolecules).

(3) Experiments with time resolution (which may reach $10 \mathrm{fs}$ in the case of XFEL) become of prime importance. Unfortunately, modern multipixel 2D detectors do not make it possible to operate at these frequencies; therefore, methods of pump-and-probe type are being actively developed.

(4) The new possibilities of detectors for analyzing correlation functions in different pixels and at different instants make it possible to analyze data of correlation functions with allowance for spatial dispersion (twotime correlations in multipixel datasets).

(5) To implement successfully interference and phase-sensitive experiments, one needs radiation sources with a high spatial and temporal coherence.

(6) The role of soft $X$ rays increases when studying biological objects and samples of modern "nonmetallic" materials science. The emphasis shifts towards the analysis of $K$-edge absorption images of light atoms $(\mathrm{C}, \mathrm{N}, \mathrm{O})$, and scattering occurs without diffraction.

The development of X-ray structural nanodiagnostics in the next decade will be determined mainly by the transition to modern megascience systems, fourthgeneration SR sources, and XFELs. It is planned to create a number of megafacilities in the Russian Federation, including the SILA source, and reconstruct the Kurchatov SR source. In view of the aforesaid, the formation and development of the research and methodical base for carrying out studies using modern CDI methods becomes an important task, which must be performed to provide competitive potential of Russian scientific community in the field of X-ray diffraction studies in the nearest future.

\section{FUNDING}

This study was supported in part by the Russian Foundation for Basic Research, project nos. 18-29-15007_mk and 19-29-12043_mk, and Ministry of Science and Higher Education of the Russian Federation within the State assignment for the Federal Scientific Research Centre "Crystallography and Photonics" of the Russian Academy of Sciences. 


\section{OPEN ACCESS}

This article is licensed under a Creative Commons Attribution 4.0 International License, which permits use, sharing, adaptation, distribution and reproduction in any medium or format, as long as you give appropriate credit to the original author(s) and the source, provide a link to the Creative Commons license, and indicate if changes were made. The images or other third party material in this article are included in the article's Creative Commons license, unless indicated otherwise in a credit line to the material. If material is not included in the article's Creative Commons license and your intended use is not permitted by statutory regulation or exceeds the permitted use, you will need to obtain permission directly from the copyright holder. To view a copy of this license, visit http://creativecommons.org/licenses/by/4.0/.

\section{ADDITIONAL INFORMATION}

The publication of this Open Access article was funded by Pleiades Publishing.

\section{REFERENCES}

1. M. V. Koval'chuk, Crystallogr. Rep. 59 (6), 779 (2014). https://doi.org/10.7868/S0023476114060150

2. M. V. Koval'chuk, Crystallogr. Rep. 56 (4), 553 (2011).

3. B. K. Vainshtein, X-Ray Diffraction from Chain Molecules (Izd-vo AN SSSR, Moscow, 1963) [in Russian].

4. B. K. Vainshtein, Zh. Eksp. Teor. Fiz. 27, 44 (1954).

5. B. K. Vainshtein, Usp. Fiz. Nauk 88 (3), 527 (1966).

6. I. P. Kuranova, Crystallogr. Rep. 46 (4), 601 (2001).

7. M. V. Koval'chuk and V. O. Popov, Nauka Ross. 195 (3), 4 (2013).

8. I. P. Kuranova and M. V. Koval'chuk, Priroda 1183 (3), 12 (2014).

9. D. Sayre, Prospects for Long-Wavelength X-Ray Microscopy and Diffraction. Imaging Processes and Coherence in Physics. Lecture Notes in Physics, Vol. 112, Ed. by M. Schlenker (Springer, Berlin, 1980). https://doi.org/10.1007/3-540-09727-9_82

10. J. Bernal and D. Crowfoot, Nature 133, 794 (1934). https://doi.org/10.1038/133794b0

11. M. M. Bluhm, G. Bodo, H. M. Dintzis, et al., Proc. Roy. Soc. A 246, 369 (1958). https://doi.org/10.1098/rspa.1958.0145

12. W. B. Yun, J. Kirz, and D. Sayre, Acta Crystallogr. A 43, 131 (1987). https://doi.org/10.1107/S0108767387099744

13. J. Miao, P. Charalambous, et al., Nature 400, 342 (1999). https://doi.org/10.1038/22498

14. J. Miao, T. Ishikawa, I. K. Robinson, et al., Science 348 (6234), 530 (2015). https://doi.org/10.1126/science.aaa 1394

15. A. P. Mancuso, O. M. Yefanov, and I. A. Vartanyants, J. Biotechnol. 149 (4), 229 (2010). https://doi.org/10.1016/j.jbiotec.2010.01.024

16. H. N. Chapman and K. A. Nugent, Nat. Photonics 4, 833 (2010).

https://doi.org/10.1038/nphoton.2010.240
17. Strategy for Scientific and Technological Development of the Russian Federation. http://static.kremlin.ru/media/acts/files/0001201612010007.pdf

18. Y. H. Lo, L. Zhao, M. Gallagher-Jones, et al., Nature Commun. 9, 1826 (2018). https://doi.org/10.1038/s41467-018-04259-9

19. https://www.esrf.fr/Apache_files/Upgrade/ESRF-orange-book.pdf

20. https://www.esrf.fr/files/live/sites/www/files/about/upgrade/documentation/Design\%20Report-reducedjan19.pdf

21. C. G. Schroer, I. Agapov, W. Brefeld, et al., J. Synchrotron Radiat. 25 (5), 1277 (2018). https://doi.org/10.1107/S1600577518008858

22. https://bib-pubdb1.desy.de/record/426140/files/DESY-PETRAIV-Conceptual-Design-Report.pdf

23. https://www.aps.anl.gov/APS-Upgrade/Documents

24. A. E. Blagov, V Mire Nauki, Nos. 1-2 (2020). https://sciam.ru/articles/details/nazad-v-budushhee

25. V. V. Lider and M. V. Koval'chuk, Crystallogr. Rep. 58, 769 (2013). https://doi.org/10.7868/S0023476113050068

26. V. V. Lider, Usp. Fiz. Nauk 185, 393 (2015). https://doi.org/10.3367/UFNr.0185.201504d.0393

27. V. G. Kohn and M. S. Folomeshkin, J. Synchrotron Radiat. 28 (2), 419 (2021).

28. V. V. Aristov, M. V. Grigoriev, S. M. Kuznetsov, et al., Appl. Phys. Lett. 77, 4058 (2000). https://doi.org/10.1063/1.1332401

29. G. S. Waldman, J. Opt. Soc. Am. 56, 215 (1966). https://doi.org/10.1364/JOSA.56.000215

30. X-Ray Optics and Microscopy, Ed. by G. Schmahl and D. M. Rudolph (Mir, Moscow, 1987) [in Russian].

31. H. Horman and H. M. Chau, Appl. Opt. 6 (2), 317 (1967). https://doi.org/10.1364/AO.6.000317

32. G. E. Ice, J. D. Budai, and J. W. L. Pang, Science 334, 1234 (2011). https://doi.org/10.1126/science.1202366

33. H. Jiang, Ch. Song, Ch. Chen, et al., Proc. Natl. Acad. Sci. U. S. A. 107, 11234 (2010). https://doi.org/10.1073/pnas.1000156107

34. J. W. Miao, K. O. Hodgson, T. Ishikawa, et al., Proc. Natl Acad. Sci. U. S. A. 100 (1), 110 (2003). https://doi.org/10.1073/pnas.232691299

35. Y. Nishino, Y. Takahashi, N. Imamoto, et al., Phys. Rev. Lett. 102018101 (2009). https://doi.org/10.1103/PhysRevLett.102.018101

36. D. Shapiro, P. Thibault, T. Beetz, et al., Proc. Natl Acad. Sci. U. S. A. 102, 15343 (2005). https://doi.org/10.1073/pnas.0503305102

37. M. Marvin Seibert, S. Boutet, M. Svenda, et al., J. Phys. B 43194015 (2010). https://doi.org/10.1088/0953-4075/43/19/194015

38. K. Giewekemeyer, P. Thibault, S. Kalbfleisch, et al., Proc. Natl. Acad. Sci. U. S. A. 107, 529 (2010). https://doi.org/10.1073/pnas.0905846107

39. C. Song, H. Jiang, A. Mancuso, et al., Phys. Rev. Lett. 101158101 (2008). https://doi.org/10.1103/PhysRevLett.101.158101

40. M. Seibert, T. Ekeberg, F. Maia, et al., Nature 470, 78 (2011).

https://doi.org/10.1038/nature09748 
41. D. Shapiro, Y. S. Yu, T. Tyliszczak, et al., Nat. Photonics 8, 765 (2014). https://doi.org/10.1038/nphoton.2014.207

42. C. Song, R. Bergstrom, D. Ramunno-Johnson, et al., Phys. Rev. Lett. 100 (2), 025504 (2008). https://doi.org/10.1103/PhysRevLett.100.025504

43. A. Tripathi, J. Mohanty, S. H. Dietze, et al., Proc. Natl. Acad. Sci. U. S. A. 108, 13393 (2011). https://doi.org/10.1073/pnas.1104304108

44. M. Newton, S. Leake, R. Harder, et al., Nat. Mater. 9, 120 (2010). https://doi.org/10.1038/nmat2607

45. H. Chapman, A. Barty, M. Bogan, et al., Nat. Phys. 2, 839 (2006). https://doi.org/10.1038/nphys461

46. P. Thibault, M. Dierolf, A. Menzel, et al., Science 321, 379 (2008). https://doi.org/10.1126/science. 1158573

47. M. Pfeifer, G. Williams, I. Vartanyants, et al., Nature 442, 63 (2006). https://doi.org/10.1038/nature04867

48. I. Robinson and R. Harder, Nat. Mater. 8, 291 (2009). https://doi.org/10.1038/nmat2400

49. I. K. Robinson, I. A. Vartanyants, G. J. Williams, et al., Phys. Rev. Lett. 87, 195505 (2001). https://doi.org/10.1103/PhysRevLett.87.195505

50. K. A. Nugent, A. G. Peele, H. M. Quiney, et al., Acta Crystallogr. A 61, 373 (2005). https://doi.org/10.1107/S010876730501055X

51. T. A. Pitts and J. F. Greenleaf, IEEE Trans. Ultrason. Ferroelectr. Freq. Control. 50, 1035 (2003). https://doi.org/10.1109/tuffc.2003.1226547

52. G. J. Williams, H. M. Quiney, B. B. Dhal, et al., Phys. Rev. Lett. 97 (2), 025506 (2006). https://doi.org/10.1103/PhysRevLett.97.025506

53. B. Abbey, K. Nugent, G. Williams, et al., Nat. Phys. 4, 394 (2008).

54. R. H. T. Bates, Optik 61, 247 (1982).

55. R. H. T. Bates, Computer Vision, Graphics, Image Process. 25 (2), 205 (1984). https://doi.org/10.1016/0734-189X(84)90103-8

56. R. H. T. Bates and M. McDonnell, Image Restoration and Reconstruction (Oxford Univ. Press, 1986).

57. R. P. Millane, J. Opt. Soc. Am. A 13, 725 (1996). https://doi.org/10.1364/JOSAA.13.000725

58. R. W. Gerchberg and O. Saxton, Optik 35, 237 (1972).

59. J. R. Fienup, Opt. Lett. 3, 27 (1978). https://doi.org/10.1364/OL.3.000027

60. J. R. Fienup, Appl. Opt. 21, 2758 (1982). https://doi.org/10.1364/AO.21.002758

61. V. Elser, J. Opt. Soc. Am. A 20, 40 (2003). https://doi.org/10.1364/JOSAA.20.000040

62. S. Marchesini, Rev. Sci. Instrum. 78 (1), 049901 (2007). https://doi.org/10.1063/1.2403783

63. C.-C. Chen, J. Miao, C. W. Wang, et al., Phys. Rev. B 76 (6), 064113 (2007). https://doi.org/10.1103/PhysRevB.76.064113

64. M. Dierolf, O. Bunk, S. Kynde, et al., Europhys. News 39 (1), 22 (2008). https://doi.org/10.1051/epn:2008003

65. F. Pfiffer, Nat. Photonics 12, 9 (2018). https://doi.org/10.1038/s41566-017-0072-5

66. W. Hoppe, Acta Crystallogr. A 25, 495 (1969). https://doi.org/10.1107/S0567739469001045
67. J. M. Rodenburg and R. H. T. Bates, Philos. Trans. R. Soc. A 339, 521 (1992).

68. P. D. Nellist, B. C. McCallum, and J. M. Rodenburg, Nature 374, 630 (1995). https://doi.org/10.1038/374630a0

69. H. N. Chapman, Ultramicroscopy 66, 153 (1996). https://doi.org/10.1016/S0304-3991(96)00084-8

70. H. M. L. Faulkner and J. M. Rodenburg, Phys. Rev. 93 (2), 023903 (2004).

https://doi.org/10.1103/PhysRevLett.93.023903

71. J. M. Rodenburg and H. M. L. Faulkner, Appl. Phys. Lett. 85, 4795 (2004).

https://doi.org/10.1063/1.1823034

72. J. M. Rodenburg, A. C. Hurst, A. G. Cullis, et al., Phys. Rev. Lett. 98 (3), 034801 (2007).

https://doi.org/10.1103/PhysRevLett.98.034801

73. P. Thibault, M. Dierolf, A. Menzel, et al., Science 321 (5887), 379 (2008).

https://doi.org/10.1063/1.3332591

74. A. Schropp, P. Boye, J. M. Feldkamp, et al., Appl. Phys. Lett. 96 (9), 091102 (2010).

https://doi.org/10.1063/1.3332591

75. P. Godard, G. Carbone, M. Allain, et al., Nat. Comm. 2, 568 (2011).

https://doi.org/10.1038/ncomms 1569

76. Y. Takahashi, N. Zettsu, Y. Nishino, et al., Nano Lett. 10, 1922 (2010).

https://doi.org/10.1021/nl100891n

77. M. Holler, M. Guizar-Sicairos, E. Tsai, et al., Nature 543, 402 (2017).

https://doi.org/10.1038/nature21698

78. K. Giewekemeyer, Thibault. P. Pierre, S. Kalbfleisch, et al., Proc. Natl. Acad. Sci. U. S. A. 107, 529 (2010. https://doi.org/10.1073/pnas.0905846107

79. M. Dierolf, A. Menzel, P. Thibault, et al., Nature 467, 436 (2010).

https://doi.org/10.1038/nature09419

80. J. M. Rodenburg, Adv. Imag. Elect. Phys. 150, 87 (2008).

https://doi.org/10.1016/S1076-5670(07)00003-1

81. E. H. R. Tsai, I. Usov, A. Diaz, et al., Opt. Express 24, 29089 (2016).

https://doi.org/10.1364/OE.24.029089

82. A. C. Kak and M. Slaney, Algorithms for Reconstruction with Nondiffracting Sources in Principles of Computerized Tomographic Imaging, Ed. by A. C. Kak (IEEE, 1989), Ch. 3 .

83. J. M. Cowley and A. F. Moodie, Acta Crystallogr. A 10, 609 (1957). https://doi.org/10.1107/S0365110X57002194

84. P. Li and A. Maiden, Sci. Rep. 8, 2049 (2018). https://doi.org/10.1038/s41598-018-20530-X

85. A. M. Maiden and J. M. Rodenburg, Ultramicroscopy 109, 1256 (2009).

https://doi.org/10.1016/j.ultramic.2009.05.012

86. A. M. Maiden, M. J. Humphry, and J. M. Rodenburg, J. Opt. Soc. Am. A 29, 1606 (2012).

https://doi.org/10.1364/JOSAA.29.001606

87. T. Sun, Z. Jiang, J. Strzalka, et al., Nat. Photonics 6, 586 (2012).

https://doi.org/10.1038/nphoton.2012.178

88. S. Marathe, S. S. Kim, S. N. Kim, et al., Opt. Express 18, 7253 (2010).

https://doi.org/10.1364/OE.18.007253 
89. S. Roy, D. Parks, K. Seu, et al., Nat. Photonics 5, 243 (2011) https://doi.org/10.1038/nphoton.2011.11

90. B. Zhang, D. F. Gardner, M. Seaberg, et al., Ultramicroscopy 158, 98 (2015).

https://doi.org/10.1016/j.ultramic.2015.07.006

91. L. G. Parratt, Phys. Rev. 95, 359 (1954). https://doi.org/10.1103/PhysRev.95.359

92. S. K. Sinha, E. B. Sirota, S. Garoff, et al., Phys. Rev. 38, 2297 (1988). https://doi.org/10.1103/PhysRevB.38.2297

93. Z. Jiang, D. R. Lee, S. Narayanan, et al., Phys. Rev. B 84 (7), 075440 (2011). https://doi.org/10.1103/PhysRevB.84.075440

94. G. Grübel and F. Zontone, J. Alloys Compd. 362 (12), 3 (2004). https://doi.org/10.1016/S0925-8388(03)00555-3

95. G. Grübel, A. Madsen, and A. Robert, X-Ray Photon Correlation Spectroscopy (XPCS) in Soft Matter Characterization, Ed. by R. Borsali and B. Pecora (Springer, Heidelberg, 2008), p. 954.

96. M. Sutton, C. R. Phys. 9 (5-6), 657 (2008). https://doi.org/10.1016/j.crhy.2007.04.008

97. S. B. Dierker, R. Pindak, R. M. Fleming, et al., Phys. Rev. Lett. 75 (3), 449 (1995). https://doi.org/10.1103/PhysRevLett.75.449

98. T. Thurn-Albrecht, W. Steffen, A. Patkowski, et al., Phys. Rev. Lett. 77 (27), 5437 (1996).

https://doi.org/10.1103/PhysRevLett.77.5437

99. L. B. Lurio, D. Lumma, A. R. Sandy, et al., Phys. Rev. Lett. 84 (4), 785 (2000).

https://doi.org/10.1103/PhysRevLett.84.785

100. D. Lumma, M. A. Borthwick, P. Falus, et al., Phys. Rev. Lett. 86 (10), 2042 (2001).

https://doi.org/10.1103/PhysRevLett.86.2042

101. S. G. J. Mochrie, A. M. Mayes, A. R. Sandy, et al., Phys. Rev. Lett. 78 (7), 1275 (1997). https://doi.org/10.1103/PhysRevLett.78.1275

102. P. Falus, M. A. Borthwick, and S. G. J. Mochrie, Phys. Rev. Lett. 94 (1), 016105 (2005). https://doi.org/10.1103/PhysRevLett.94.016105

103. C. Caronna, Y. Chushkin, A. Madsen, et al., Phys. Rev. Lett. 100 (5), 055702 (2008). https://doi.org/10.1103/PhysRevLett.100.055702

104. O. G. Shpyrko, E. D. Isaacs, J. M. Logan, et al., Nature 447 (7140), 68 (2007). https://doi.org/10.1038/nature05776

105. S. Brauer, G. B. Stephenson, M. Sutton, et al., Phys. Rev. Lett. 74 (11), 2010 (1995). https://doi.org/10.1103/PhysRevLett.74.2010

106. M. Leitner, B. Sepiol, L.-M. Stadler, et al., Nat. Mater. 8 (9), 717 (2009). https://doi.org/10.1038/nmat2506

107. S. B. Dieker, R. Pindak, R. M. Fleming, et al., Phys. Rev. Lett. 75, 449 (1995). https://doi.org/10.1103/PhysRevLett.75.449

108. S. G. J. Mochrie, A. M. Mayes, A. R. Sandy, et al., Phys. Rev. Lett. 78, 1275 (1997). https://doi.org/10.1103/PhysRevLett.78.1275

109. S. Brauer, G. B. Stephenson, M. Sutton, et al., Phys. Rev. Lett. 74, 2010 (1995). https://doi.org/10.1103/PhysRevLett.74.2010

110. Z. H. Cai, B. Lai, W. B. Yun, et al., Phys. Rev. Lett. 73, $82(1994)$.

https://doi.org/10.1103/PhysRevLett.73.82
111. A. Madsen, A. Fluerasu, and B. Ruta, Synchrotron Light Sources and Free-Electron Lasers, Ed. by E. Jaeschke et al. (Springer, Cham, 2018), p. 1617. https://doi.org/10.1007/978-3-319-14394-1_29

112. B. J. Berne and R. Pecora, Dynamic Light Scattering with Application to Chemistry, Biology, and Physics (Dover Publications, 2000).

113. A. Fluerasu, A. Moussaid, A. Madsen, et al., Phys. Rev. E 76, 0100401(R) (2007).

https://doi.org/10.1103/PhysRevE.76.010401

114. I. Inoue, Y. Shinohara, A. Watanabe, et al., Opt. Express 20, 26878 (2012).

https://doi.org/10.1364/OE.20.026878

115. C. DeCaro, V. N. Karunaratne, S. Bera, et al., J. Synchrotron Radiat. 20, 332 (2013).

https://doi.org/10.1107/S0909049512051825

116. L. Li, P. Kwas'niewski, D. Orsi, et al., J. Synchrotron Radiat. 21, 1288 (2014). https://doi.org/10.1107/S1600577514015847

117. J. Verwohlt, M. Reiser, L. Randolph, et al., Phys. Rev. Lett. 120, 168001 (2018). https://doi.org/10.1103/PhysRevLett.120.168001

118. J. Möller, M. Reiser, J. Hallmann, et al., J. Synchrotron Radiat. 26, 1705 (2019). https://doi.org/10.1107/S1600577519006349

119. C. Gutt, L.-M. Stadler, A. Duri, et al., Opt. Express 17, 55 (2009). https://doi.org/10.1364/OE.17.000055

120. G. B. Stephenson, A. Robert, and G. Grübel, Nat. Mater. 8, 702 (2009). https://doi.org/10.1038/nmat2521

121. P. Emma, R. Akre, et al., Nat. Photonics 4, 641 (2010). https://doi.org/10.1038/nphoton.2010.176

122. M. Altarelli, Nucl. Instrum. Methods Phys. Res. B 269, 2845 (2011). https://doi.org/10.1016/j.nimb.2011.04.034

123. T. Ishikawa, H. Aoyagi, T. Asaka, et al., Nat. Photonics 6, 540 (2012). https://doi.org/10.1038/nphoton.2012.141

124. H.-S. Kang, C.-K. Min, H. Heo, et al., Nat. Photonics 11, 708 (2017). https://doi.org/10.1038/s41566-017-0029-8

125. C. Milne, T. Schietinger, M. Aiba, et al., Appl. Sci. 7, 720 (2017). https://doi.org/10.3390/app7070720

126. Z. Kam, Macromolecules 10, 927 (1977). https://doi.org/10.1021/ma60059a009

127. N. A. Clark, B. J. Ackerson, and A. J. Hurd, Phys. Rev. Lett. 50, 1459 (1983).

128. B. J. Ackerson and N. A. Clark, Chem. Soc. 76, 219 (1983).

129. P. Wochner, C. Gutt, T. Autenrieth, et al., Proc. Natl. Acad. Sci. U. S. A 106, 11511 (2009). https://doi.org/10.1073/pnas.0905337106

130. M. Altarelli, R. P. Kurta, and I. A. Vartanyants, Phys. Rev. B 82, 104207 (2010). https://doi.org/10.1103/PhysRevB.82.104207

131. R. P. Kurta, M. Altarelli, E. Weckert, et al., Phys. Rev. B 85, 184204 (2012). https://doi.org/10.1103/PhysRevB.85.184204

132. F. Lehmkühler, G. Grübel, and C. Gutt, J. Appl. Crystallogr. 47, 1315 (2014).

https://doi.org/10.1107/S1600576714012424 
133. T. Latychevskaia, G. F. Mancini, and F. Carbone, Sci. Rep. 5, 16573 (2015). https://doi.org/10.1038/srep 16573

134. M. A. Schroer, F. Westermeier, F. Lehmkühler, et al., J. Chem. Phys. 144, 084903 (2016). https://doi.org/10.1063/1.4941563

135. M. A. Schroer, C. Gutt, and G. Grübel, Phys. Rev. E 90, 012309 (2014). https://doi.org/10.1103/PhysRevE.90.012309

136. I. A. Zaluzhnyy, R. P. Kurta, E. A. Sulyanova, et al., Soft Matter 13, 3240 (2017). https://doi.org/10.1039/C7SM00343A

137. I. Zaluzhnyy, R. P. Kurta, A. André, et al., Nano Lett. 17 (6), 3511 (2017). https://doi.org/10.1021/acs.nanolett.7b00584

138. R. P. Kurta, L. Grodd, E. Mikayelyan, et al., Phys. Chem. Chem. Phys. 17, 7404 (2015). https://doi.org/10.1039/C5CP00426H

139. K. A. Seu, S. Roy, R. Su, et al., Appl. Phys. Lett. 98, 122505 (2011). https://doi.org/10.1063/1.3569952

140. D. Starodub, A. Aquila, S. Bajt, et al., Nat. Commun. 3, 1276 (2012). https://doi.org/10.1038/ncomms2288

141. B. Pedrini, A. Menzel, M. Guizar-Sicairos, et al., Nat. Commun. 4, 1647 (2013). https://doi.org/10.1038/ncomms2622

142. D. Sheyfer, Ph. D. Thesis (Universitat Hamburg, 2017).

143. A. Madsen, J. Hallmann, G. Ansaldi, et al., J. Synchrotron Radiat. 28 (2), 637 (2021). https://doi.org/10.1107/S1600577521001302

144. K. Ayyer, L. Xavier, J. Bielecki, et al., Optica 8, 15 (2021). https://doi.org/10.1364/OPTICA.410851

145. T. Mandl, C. Östlin, I. E. Dawod, et al., J. Phys. Chem. Lett. 11 (15), 6077 (2020). https://doi.org/10.1021/acs.jpclett.0c01144

146. M. O. Wiedorn, D. Oberthür, R. Bean, et al., Nat. Commun. 9 (1), 4025 (2018). https://doi.org/10.1038/s41467-018-06156-7

147. N. Mukharamova, S. Lazarev, J.-M. Meijer, et al., Sci. Rep. 10 (1), 10780 (2020). https://doi.org/10.1038/s41598-020-67214-z

148. D. Rupp, L. Flückiger, M. Adolph, et al., Struct. Dyn. 7 (3), 034303 (2020).

https://doi.org/10.1063/4.0000006

149. E. Sobolev, S. Zolotarev, K. Giewekemeyer, et al., Commun. Phys. 3, 97 (2020). https://doi.org/10.1038/s42005-020-0362-y

150. J. Bielecki, F. R. N. C. Maia, A. P. Mancuso, et al., Struct. Dyn. 7 (4), 040901 (2020). https://doi.org/10.1063/4.0000024

151. J. Möller, M. Sprung, A. Madsen, et al., IUCrJ 6 (5), 1 (2019). https://doi.org/10.1107/S2052252519008273

152. V. Kärcher, S. Roling, L. Samoylova, et al., J. Synchrotron Radiat. 28 (1), 350 (2021). https://doi.org/10.1107/S1600577520014563

153. M. Seaberg, R. Cojocaru, S. Berujon, et al., J. Synchrotron Radiat. 26 (4), xl5031 (2019). https://doi.org/10.1107/S1600577519005721
154. M. Polikarpov, G. Bourenkov, I. Snigireva, et al., Acta Crystallogr. D 75 (11), 947 (2019). https://doi.org/10.1107/S2059798319011379

155. J. Hagemann, M. Vassholz, H. Hoeppe, et al., J. Synchrotron Radiat. 28 (1), 52 (2021). https://doi.org/10.1107/S160057752001557X

156. M. Makita, G. Seniutinas, M. H. Seaberg, et al., Optica 7 (5), 404 (2020). https://doi.org/10.1364/OPTICA.390601

157. C. David, S. Gorelick, S. Rutishauser, et al., Sci. Rep. 1, 57 (2011). https://doi.org/10.1038/srep00057

158. B. Lengeler, C. G. Schroer, M. Kuhlmann, et al., J. Phys. D 38, A218 (2005). https://doi.org/10.1088/0022-3727/38/10A/042

159. H. N. Chapman, C. Caleman, and N. Timneanu, Philos. Trans. R. Soc. B 369, 20130313 (2014). https://doi.org/10.1098/rstb.2013.0313

160. A. Allahgholi, J. Becker, A. Delfs, et al., J. Synchrotron Radiat. 26 (1), 74 (2019). https://doi.org/10.1107/S1600577518016077

161. W. Lu, B. Friedrich, T. Noll, et al., Rev. Sci. Instrum. 89, 063121 (2018). https://doi.org/10.1063/1.5027071

162. H. Li, Y. Sun, M. Sutton, et al., Opt. Lett. 45, 2086 (2020). https://doi.org/10.1364/OL.389977

163. Y. Sun, N. Wang, S. Song, et al., Opt. Lett. 44, 2582 (2019). https://doi.org/10.1364/OL.44.002582

164. T. Weitkamp, A. Diaz, C. David, et al., Opt. Express 13, 6296 (2005). https://doi.org/10.1364/OPEX.13.006296

165. S. Sala, B. J. Daurer, M. Odstrcil, et al., J. Appl. Crystallogr. 53, 949 (2020). https://doi.org/10.1107/S1600576720006913

166. M. Hirose, T. Higashino, N. Ishiguro, et al., Opt. Express 28, 1216 (2020). https://doi.org/10.1364/OE.378083

167. E. Sobolev, S. Zolotarev, K. Giewekemeyer, et al., Commun. Phys. 3, 97 (2019). https://doi.org/10.1038/s42005-020-0362-y

168. I. Robinson, J. Schwenke, M. Yusuf, et al., J. Phys. B 48, 244007 (2015). https://doi.org/10.1088/0953-4075/48/24/244007

169. F. Seiboth, L. B. Fletcher, D. McGonegle, et al., Appl. Phys. Lett. 112, 221907 (2018). https://doi.org/10.1063/1.5031907

170. P. Vagovic, T. Sato, L. Mikes, et al., Optica 6, 1106 (2019). https://doi.org/10.1364/OPTICA.6.001106

171. G. Kastirke, M. S. Schöffler, M. Weller, et al., Phys. Rev. X 10 (2), 021052 (2020). https://doi.org/10.1103/PhysRevX.10.021052

172. U. Eichmann, H. Rottke, S. Meise, et al., Science 369 (6511), 1630 (2020). https://doi.org/10.1126/science.abc2622

173. S. Serkez, A. Trebushinin, M. Veremchuk, et al., Phys. Rev. Accel. Beams. 22 (11), 110705 (2019). https://doi.org/10.1103/PhysRevAccelBeams.22.110705

Translated by Yu. Sin'kov 